\title{
Quantum Optics Properties of QCD Vacuum
}

\author{
V. Kuvshinov ${ }^{1, a}$, V. Shaparau ${ }^{1, b}$, E. Bagashov ${ }^{1, c}$ \\ ${ }^{1}$ Joint Institute for Power and Nuclear Research - Sosny \\ National Academy of Science of Belarus \\ PO box 119, 220109 Minsk, BELARUS
}

\begin{abstract}
Theoretical justification of the occurrence of multimode squeezed and entangled colour states in QCD is given. We show that gluon entangled states which are closely related with corresponding squeezed states can appear by the four-gluon self-interaction. Correlations for the collinear gluons are revealed two groups of the colour correlations which is significant at consider of the quark-antiquark pair productions.

It is shown that the interaction of colour quark with the stochastic vacuum of QCD leads to the loss of information on the initial colour state of the particle, which gives a new perspective regarding the confinement of quarks phenomenon. The effect is demonstrated for a single particle and in the multiparticle case is proposed.

Quantum characteristics (purity and von Neumann entropy) are used to analyse the process of interaction.
\end{abstract}

\section{Introduction}

Many experiments at $e^{+} e^{-}, p \bar{p}, e p$ colliders are devoted to hadronic jet physics, since detailed studies of jets are important for better understanding and testing both perturbative and non-perturbative QCD and also for finding manifestations of new physics. Although the nature of jets is of a universal character, $e^{+} e^{-}$- annihilation stands out among hard processes, since jet events admit a straightforward and clear-cut separation in this process.

In the reaction $e^{+} e^{-} \rightarrow$ hadron four evolution phases are recognized at various time and space scales (Fig.1). These are (I) the production of a quark-antiquark pair: $e^{+} e^{-} \rightarrow q \bar{q}$; (II) the emissions of gluons from primary partons - perturbative evolution of the quark-gluon cascade; (III) the non-perturbative evolution and the hadronization of quarks and gluons; (IV) the decays of unstable particles.

The second phase of $e^{+} e^{-}$- annihilation has been well understood and sufficiently accurate predictions for it have been obtained within the perturbative QCD (PQCD) [1]. But predictions of the PQCD are limited by small effective coupling $\alpha\left(Q^{2}\right)<1$ and third phase is usually taken into account either through a constant factor which relates partonic features with hadronic ones (within local partonhadron duality) or through the application of various phenomenological models of hadronization. As a consequence, theoretical predictions for jet characteristics remain unsatisfactory. For example, the

\footnotetext{
ae-mail: v.kuvshinov@sosny.bas-net.by

be-mail: shaparau@sosny.bas-net.by

ce-mail: bagashov@sosny.bas-net.by
} 
width of the multiplicity distribution (MD) according to the predictions of PQCD is larger than the experimental one. The discrepancies between theoretical calculations and experimental data suggest that the non-perturbative evolution of the quark-gluon cascade plays important role. New gluon states, generated at the non-perturbative stage, contribute to various features of jets. In particular, such a contribution to MD can be made in the form of the sub-Poissonian distribution $[2,3]$. Therefore we must take into account both perturbative and non-perturbative stage of the jet evolution.

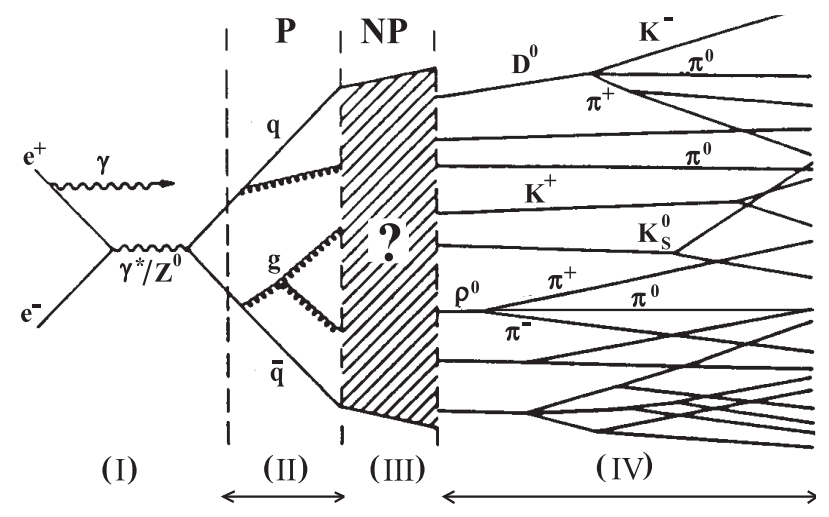

Figure 1. Jet evolution

It is known that such property is inherent for the squeezed states (SS), which are well studied in quantum optics (QO) [4]-[6]. Squeezed states have uncommon properties: they display a specific behaviour of the factorial and cumulant moments [7] and can have both sub-Poissonian and superPoissonian statistics corresponding to antibunching and bunching of photons. Moreover oscillatory behaviour MD of photon SS is differentiated from Poissonian and Negative binomial distributions (NBD).

Therefore we believe that the non-perturbative stage of gluon evolution can be one of sources of the gluon SS in QCD by analogy with nonlinear medium for photon SS in QO. Gluon MD in the range of the small transverse momenta (thin ring of jet) is Poissonian [8]. Quark-gluon MD in the whole jet at the end of the perturbative cascade can be represented as a combination of Poissonian distributions each of which corresponds to a coherent state. Studying a further evolution of gluon states at the non-perturbative stage of jet evolution we obtain new gluon states. These states are formed as a result of non-perturbative self-interaction of the gluons expressed by nonlinearities of Hamiltonian. Using the Local parton hadron duality it is easy to show that in this case behaviour of hadron multiplicity distribution in jet events is differentiated from the negative binomial one that is confirmed by experiments for $p p, p \bar{p}$-collisions [9]-[11].

At finite squeezed $r$ (continuous variable) entangled state is known from quantum optics as a two-mode squeezed state $[4,18]$

$$
|\mathrm{f}\rangle=\hat{S}_{12}(r)|0\rangle_{1}|0\rangle_{2}=\frac{1}{\cosh r} \sum_{n=0}^{\infty}(\tanh r)^{n}|n\rangle_{1}|n\rangle_{2},
$$

where $\hat{S}_{12}(r)=\exp \left\{r\left(\hat{a}_{1}^{+} \hat{a}_{2}^{+}-\hat{a}_{1} \hat{a}_{2}\right)\right\}$ is operator of two-mode squeezing. It is not difficult to demonstrate that the state vector $|\mathrm{f}\rangle$ describes the entangled state. Each of these entangled states has a uncommon property: a measurement over one particle have an instantaneous effect on the other, possibly located at a large distance. 
The dimensionless coefficient

$$
y=\left[\frac{\left|\overline{\hat{a}_{1} \hat{a}_{2}^{+}}\right|^{2}+\left|\overline{\hat{a}_{1} \hat{a}_{2}}\right|^{2}}{2\left(\overline{\hat{a}_{1}^{+} \hat{a}_{1}}+1 / 2\right)\left(\overline{\hat{a}_{2}^{+} \hat{a}_{2}}+1 / 2\right)}\right]^{1 / 2}
$$

is the measure of entanglement for two-mode states [19], $0 \leq y<1$ (entanglement is not observed when $y=0$ ). Here $\overline{\hat{a}}_{i} \hat{a}_{j}^{+}=\left\langle\hat{a}_{i} \hat{a}_{j}^{+}\right\rangle-\left\langle\hat{a}_{i}\right\rangle\left\langle\hat{a}_{j}^{+}\right\rangle, \hat{a}_{i}, \hat{a}_{j}^{+}$are the annihilation and creation operators correspondingly. Averaging the annihilation and creation operators in the expression (2) over the vector $|\mathrm{f}\rangle$ (1) at small squeeze factor we have

$$
y \simeq \sqrt{2} r .
$$

Two-mode gluon states with two different colours can lead to $q \bar{q}$-entangled states. Interaction of the quark entangled states with stochastic vacuum (quantum measurement) has a remarkable property, namely, as soon as some measurement projects one quark onto a state with definite colour, the other quark also immediately obtains opposite colour that leads to coupling of quark-antiquark pair, string tension inside $q \bar{q}$-pair and free propagation of colourless hadrons. Therefore the investigation of the gluon entangled states connected with the corresponding squeezed ones is the issue of the day.

\section{Multimode squeezed states of the gluons}

By analogy with QO [6] a multimode squeezing condition for gluons with different colors $i_{1}, \ldots, i_{p}$ is written as

$$
\left.\hat{N}\left(\Delta\left(\hat{X}_{\lambda}^{i_{1}, \ldots, i_{p}}\right)_{2}\right)^{2}\right\rangle<0,
$$

where $\Delta\left(\hat{X}_{\lambda}^{i_{1}, \ldots, i_{p}}\right)=\hat{X}_{\lambda}^{i_{1}, \ldots, i_{p}}-\left\langle\hat{X}_{\lambda}^{i_{1}, \ldots, i_{p}}\right\rangle, \hat{N}$ is a normal ordering operator, the phase-sensitive Hermitian operators $\left(\hat{X}_{\lambda}^{i_{1}, \ldots, i_{p}}\right)_{1}=\frac{1}{2 \sqrt{p}} \sum_{j=1}^{p}\left[\hat{b}_{\lambda}^{i_{j}}+\left(\hat{b}_{\lambda}^{i_{j}}\right)^{+}\right]$and $\left(\hat{X}_{\lambda}^{i_{1}, \ldots, i_{p}}\right)_{2}=\frac{1}{2 i \sqrt{p}} \sum_{j=1}^{p}\left[\hat{b}_{\lambda}^{i_{j}}-\left(\hat{b}_{\lambda}^{i_{j}}\right)^{+}\right]$are linear combination of the annihilating (creating) operators $\hat{b}_{\lambda}^{i_{j}}\left(\hat{b}_{\lambda}^{i_{j}+}\right), i_{1}, \ldots, i_{p}=\overline{1,8}$ are gluon color charges, $\lambda$ is a polarization index. Averaging in (4) is performed over final state vector

$$
\left.|\mathrm{f}\rangle \simeq \mid \text { in }\rangle-i t\left(\hat{H}_{\mathrm{I}}^{(3)}(0)+\hat{H}_{\mathrm{I}}^{(4)}(0)\right) \mid \text { in }\right\rangle
$$

which describes gluon system later small time $t$. Operators $\hat{H}_{\mathrm{I}}^{(3)}(t)$ and $\hat{H}_{\mathrm{I}}^{(4)}(t)$ describing the three- and four-gluon selfinteractions include combinations of three and four annihilating and creating operators [20].

$$
\begin{aligned}
\hat{H}_{\mathrm{I}}^{(3)}( & ()=i \frac{g}{(2 \pi)^{3 / 2}} f_{a b c} \sum_{\lambda_{1}, \lambda_{2}, \lambda_{3}} \int d \tilde{k}_{1} d \tilde{k}_{2} d \tilde{k}_{3} \times\left\{\vec{k}_{1} \vec{\varepsilon}_{\lambda_{2}}\left(k_{2}\right) \varepsilon_{\lambda_{1}}^{v}\left(k_{1}\right) \varepsilon_{v}^{\lambda_{3}}\left(k_{3}\right) \delta\left(\vec{k}_{1}+\vec{k}_{2}+\vec{k}_{3}\right)\right. \\
\times & {\left[\hat{b}_{\lambda_{1}}^{a}\left(k_{1}\right) \hat{b}_{\lambda_{2}}^{b}\left(k_{2}\right) \hat{b}_{\lambda_{3}}^{c}\left(k_{3}\right) e^{-2 i\left(k_{01}+k_{02}+k_{03}\right) t}-\hat{b}_{\lambda_{1}}^{a+}\left(k_{1}\right) \hat{b}_{\lambda_{2}}^{b+}\left(k_{2}\right) \hat{b}_{\lambda_{3}}^{c+}\left(k_{3}\right) e^{2 i\left(k_{01}+k_{02}+k_{03}\right) t}\right] } \\
& +\hat{b}_{\lambda_{1}}^{a+}\left(k_{1}\right) \hat{b}_{\lambda_{2}}^{b}\left(k_{2}\right) \hat{b}_{\lambda_{3}}^{c}\left(k_{3}\right) e^{2 i\left(k_{01}-k_{02}-k_{03}\right) t} \delta\left(\vec{k}_{1}-\vec{k}_{2}-\vec{k}_{3}\right) \\
& \times\left[\vec{k}_{3} \vec{\varepsilon}_{\lambda_{1}}\left(k_{1}\right) \varepsilon_{\lambda_{2}}^{v}\left(k_{2}\right) \varepsilon_{v}^{\lambda_{3}}\left(k_{3}\right)+\vec{k}_{2} \vec{\varepsilon}_{\lambda_{3}}\left(k_{3}\right) \varepsilon_{\lambda_{1}}^{v}\left(k_{1}\right) \varepsilon_{v}^{\lambda_{2}}\left(k_{2}\right)-\vec{k}_{1} \vec{\varepsilon}_{\lambda_{2}}\left(k_{2}\right) \varepsilon_{\lambda_{1}}^{v}\left(k_{1}\right) \varepsilon_{v}^{\lambda_{3}}\left(k_{3}\right)\right] \\
& +\hat{b}_{\lambda_{1}}^{a+}\left(k_{1}\right) \hat{b}_{\lambda_{2}}^{b+}\left(k_{2}\right) \hat{b}_{\lambda_{3}}^{c}\left(k_{3}\right) e^{2 i\left(k_{01}+k_{02}-k_{03}\right) t} \delta\left(\vec{k}_{1}+\vec{k}_{2}-\vec{k}_{3}\right) \\
& \left.\times\left[\vec{k}_{1} \vec{\varepsilon}_{\lambda_{3}}\left(k_{3}\right) \varepsilon_{\lambda_{1}}^{v}\left(k_{1}\right) \varepsilon_{v}^{\lambda_{2}}\left(k_{2}\right)+\vec{k}_{3} \vec{\varepsilon}_{\lambda_{1}}\left(k_{1}\right) \varepsilon_{\lambda_{2}}^{v}\left(k_{2}\right) \varepsilon_{v}^{\lambda_{3}}\left(k_{3}\right)-\vec{k}_{1} \vec{\varepsilon}_{\lambda_{2}}\left(k_{2}\right) \varepsilon_{\lambda_{1}}^{v}\left(k_{1}\right) \varepsilon_{v}^{\lambda_{3}}\left(k_{3}\right)\right]\right\}
\end{aligned}
$$




$$
\begin{aligned}
\hat{H}_{\mathrm{I}}^{(4)}(t) & =\frac{g^{2}}{4(2 \pi)^{3}} \sum_{\lambda_{1}, \lambda_{2}, \lambda_{3}, \lambda_{4}} \int d \tilde{k}_{1} d \tilde{k}_{2} d \tilde{k}_{3} d \tilde{k}_{4}\left\{\varepsilon_{\lambda_{1}}^{\mu}\left(k_{1}\right) \varepsilon_{\mu}^{\lambda_{3}}\left(k_{3}\right) \varepsilon_{\lambda_{2}}^{v}\left(k_{2}\right) \varepsilon_{v}^{\lambda_{4}}\left(k_{4}\right) f_{a b c} f_{a d e}\right. \\
\times & {\left[\delta ( \vec { k } _ { 1 } + \vec { k } _ { 2 } + \vec { k } _ { 3 } + \vec { k } _ { 4 } ) \left(\hat{b}_{\lambda_{1}}^{b}\left(k_{1}\right) \hat{b}_{\lambda_{2}}^{c}\left(k_{2}\right) \hat{b}_{\lambda_{3}}^{d}\left(k_{3}\right) \hat{b}_{\lambda_{4}}^{e}\left(k_{4}\right) e^{-2 i\left(k_{01}+k_{02}+k_{03}+k_{04}\right) t}\right.\right.} \\
+ & \left.\hat{b}_{\lambda_{1}}^{b+}\left(k_{1}\right) \hat{b}_{\lambda_{2}}^{c+}\left(k_{2}\right) \hat{b}_{\lambda_{3}}^{d+}\left(k_{3}\right) \hat{b}_{\lambda_{4}}^{e+}\left(k_{4}\right) e^{2 i\left(k_{01}+k_{02}+k_{03}+k_{04}\right) t}\right) \\
+ & 4 \hat{b}_{\lambda_{1}}^{b+}\left(k_{1}\right) \hat{b}_{\lambda_{2}}^{c}\left(k_{2}\right) \hat{b}_{\lambda_{3}}^{d}\left(k_{3}\right) \hat{b}_{\lambda_{4}}^{e}\left(k_{4}\right) e^{2 i\left(k_{01}-k_{02}-k_{03}-k_{04}\right) t} \delta\left(\vec{k}_{1}-\vec{k}_{2}-\vec{k}_{3}-\vec{k}_{4}\right) \\
+ & \left.4 \hat{b}_{\lambda_{1}}^{b+}\left(k_{1}\right) \hat{b}_{\lambda_{2}}^{c+}\left(k_{2}\right) \hat{b}_{\lambda_{3}}^{d+}\left(k_{3}\right) \hat{b}_{\lambda_{4}}^{e}\left(k_{4}\right) e^{2 i\left(k_{01}+k_{02}+k_{03}-k_{04}\right) t} \delta\left(\vec{k}_{1}+\vec{k}_{2}+\vec{k}_{3}-\vec{k}_{4}\right)\right] \\
+ & 2 \hat{b}_{\lambda_{1}}^{b+}\left(k_{1}\right) \hat{b}_{\lambda_{2}}^{c+}\left(k_{2}\right) \hat{b}_{\lambda_{3}}^{d}\left(k_{3}\right) \hat{b}_{\lambda_{4}}^{e}\left(k_{4}\right) e^{2 i\left(k_{01}+k_{02}-k_{03}-k_{04}\right) t} \delta\left(\vec{k}_{1}+\vec{k}_{2}-\vec{k}_{3}-\vec{k}_{4}\right) \\
\times & {\left[\varepsilon_{\lambda_{1}}^{\mu}\left(k_{1}\right) \varepsilon_{\mu}^{\lambda_{3}}\left(k_{3}\right) \varepsilon_{\lambda_{2}}^{v}\left(k_{2}\right) \varepsilon_{v}^{\lambda_{4}}\left(k_{4}\right)\left(f_{a b c} f_{a d e}+f_{a b e} f_{a d c}\right)\right.} \\
& \left.\left.+\varepsilon_{\lambda_{1}}^{\mu}\left(k_{1}\right) \varepsilon_{\mu}^{\lambda_{2}}\left(k_{2}\right) \varepsilon_{\lambda_{3}}^{v}\left(k_{3}\right) \varepsilon_{v}^{\lambda_{4}}\left(k_{4}\right) f_{a b d} f_{a c e}\right]\right\} .
\end{aligned}
$$

Here $g$ is a self-interaction constant, $d \tilde{k}=\frac{d^{3} k}{\sqrt{2 k_{0}}}, k_{0}$ is a gluon energy, $\varepsilon_{\lambda}^{\mu}$ is a polarization vector, $f_{a h b}$ are structure constants of $\mathrm{SU}_{\mathrm{c}}(3)$ group.

Initial state vector |in $\rangle$ describes gluon system at end of perturbative stage [8] and is product of the coherent states of the gluons with different colours and polarization indexes

$$
\mid \text { in }\rangle \equiv|\alpha\rangle=\prod_{\lambda=1}^{3} \prod_{b=1}^{8}\left|\alpha_{\lambda}^{b}\right\rangle .
$$

By analogy with QO gluon coherent state vector $\left|\alpha_{\lambda}^{b}\right\rangle$ is the eigenvector of the corresponding annihilation operator $\hat{b}_{\lambda}^{b}$ with the eigenvalue $\alpha_{\lambda_{1}}^{b}$ which can be written in terms of the gluon coherent field amplitude $\left|\alpha_{\lambda_{1}}^{b}\right|$ and phase $\gamma_{\lambda}^{b}$ of the given gluon field $\alpha_{\lambda_{1}}^{b}=\left|\alpha_{\lambda_{1}}^{b}\right| e^{i \gamma_{\lambda_{1}}^{b}}$. In each gluon coherent state $\left|\alpha_{\lambda}^{b}\right\rangle$ the gluon number with fixed colour $\mathrm{b}$ and polarization $\lambda$ is arbitrary (the average multiplicity of given gluon is equal to square of the gluon coherent field amplitude $\left\langle n_{\lambda}^{b}\right\rangle=\left|\alpha_{\lambda}^{b}\right|^{2}$ ) and phase of considering state $\gamma_{\lambda}^{b}$ is fixed.

Averaging the annihilation and creation operators $\hat{b}_{\lambda}^{i_{j}}, \hat{b}_{\lambda}^{i_{j}+}$ in (4) over the evolved vector $|\mathrm{f}\rangle$ which is defined according to (5) and taking into account of chosen initial state vector we write the multimode squeezing condition in the form

$$
\left\langle\hat{N}\left(\Delta\left(\hat{X}_{\lambda}^{i_{1}, \ldots, i_{p}}\right)_{2}\right)^{2}\right\rangle=\mp \frac{i t}{4 p} \sum_{j, k=1}^{p}\left\{\left\langle\alpha\left|\left[\hat{b}_{\lambda}^{i_{k}},\left[\hat{b}_{\lambda}^{i_{j}}, \hat{H}_{\mathrm{I}}(0)\right]\right]\right| \alpha\right\rangle-\left\langle\alpha\left|\left[\left[\hat{H}_{\mathrm{I}}(0), \hat{b}_{\lambda}^{i_{j}+}\right], \hat{b}_{\lambda}^{i_{k}+}\right]\right| \alpha\right\rangle\right\}<0,
$$

where $\hat{H}_{\mathrm{I}}(0)=\hat{H}_{\mathrm{I}}^{(3)}(0)+\hat{H}_{\mathrm{I}}^{(4)}(0)$.

It can be shown that only the four-gluon self-interaction can yield the multimode squeezing effect since

$$
\left[\left[\hat{H}_{\mathrm{I}}^{(3)}(0), \hat{b}_{\lambda}^{i_{j}+}\right], \hat{b}_{\lambda}^{i_{k}+}\right]=0, \quad\left[\hat{b}_{\lambda}^{i_{k}},\left[\hat{b}_{\lambda}^{i_{j}}, \hat{H}_{\mathrm{I}}^{(3)}(0)\right]\right]=0
$$


Indeed, the multimode squeezing condition can be written in the explicit form as

$$
\begin{aligned}
& \left\langle\hat{N}\left(\Delta\left(\hat{X}_{\lambda}^{i_{1}, \ldots, i_{p}}\right)_{\frac{1}{2}}\right)^{2}\right\rangle= \pm \frac{i t}{8 p k_{0}} \frac{g^{2}}{(2 \pi)^{3}} \sum_{j, k=1}^{p} \sum_{\lambda_{1}, \lambda_{2}=1}^{3} \int \prod_{i=1}^{2} \frac{d^{3} k_{i}}{\sqrt{2 k_{0 i}}} \\
& \times\left[\left(\varepsilon_{\mu}^{\lambda_{1}}\left(k_{1}\right) \varepsilon_{\lambda_{2}}^{\mu}\left(k_{2}\right)\right)\left(\varepsilon_{v}^{\lambda}(k) \varepsilon_{\lambda}^{v}(k)\right)-\left(\varepsilon_{\mu}^{\lambda_{1}}\left(k_{1}\right) \varepsilon_{\lambda}^{\mu}(k)\right)\left(\varepsilon_{v}^{\lambda_{2}}\left(k_{2}\right) \varepsilon_{\lambda}^{v}(k)\right)\right] \\
& \times\left\{f_{a i_{j} b} f_{a i_{k} c}\left[\left\langle\alpha\left|\hat{b}_{\lambda_{1}}^{b+}\left(k_{1}\right) \hat{b}_{\lambda_{2}}^{c+}\left(k_{2}\right)\right| \alpha\right\rangle-\left\langle\alpha\left|\hat{b}_{\lambda_{1}}^{b}\left(k_{1}\right) \hat{b}_{\lambda_{2}}^{c}\left(k_{2}\right)\right| \alpha\right\rangle\right]\right. \\
& \times\left[\delta\left(2 \vec{k}-\overrightarrow{k_{1}}-\overrightarrow{k_{2}}\right)-\delta\left(2 \vec{k}+\overrightarrow{k_{1}}+\overrightarrow{k_{2}}\right)\right]+\left(f_{a i_{j} b} f_{a i_{k} c}+f_{a i_{k} b} f_{a i_{j} c}\right) \\
& \left.\times\left\langle\alpha\left|\hat{b}_{\lambda_{1}}^{b+}\left(k_{1}\right) \hat{b}_{\lambda_{2}}^{c}\left(k_{2}\right)\right| \alpha\right\rangle\left[\delta\left(2 \vec{k}-\overrightarrow{k_{1}}+\overrightarrow{k_{2}}\right)-\delta\left(2 \vec{k}+\overrightarrow{k_{1}}-\overrightarrow{k_{2}}\right)\right]\right\}<0 .
\end{aligned}
$$

In particular for the collinear gluons we have the condition

$$
\left\langle\hat{N}\left(\Delta\left(X_{\lambda}^{i_{1}, \ldots, i_{p}}\right)_{2}\right)^{2}\right\rangle= \pm \frac{g^{2} t}{8 p(2 \pi)^{3} k_{0}^{2}} \sum_{j, k=1}^{p} \sum_{\lambda_{1} \neq \lambda} f_{a i_{k} b} f_{a i_{j} c}\left|\alpha_{\lambda_{1}}^{b} \| \alpha_{\lambda_{1}}^{c}\right| \sin \left(\gamma_{\lambda_{1}}^{b}+\gamma_{\lambda_{1}}^{c}\right)<0,
$$

Here $\left|\alpha_{\lambda_{1}}^{b}\right|$ and $\gamma_{\lambda_{1}}^{b}$ are an amplitude and a phase of the initial gluon coherent field, $f_{a h b}$ is a structure constant of the color group $S U_{\mathrm{c}}(3)$. The multimode squeezing condition (12) is fulfilled for any cases apart from if all initial gluon coherent fields are real or imaginary. Obviously, the larger are both the amplitudes of the initial gluon coherent fields with different colour and polarization indexes and coupling constant, the larger is multimode squeezing effect.

Thus non-perturbative four-gluon selfinteraction is source of the multimode squeezing effect.

\section{Gluon entangled states}

One of entangled condition is

$$
0<y<1,
$$

where entangled measure is defined by dimentionless value (2)

$$
y=\left[\frac{\left|\overline{\hat{b}_{\lambda}^{h} \hat{b}_{\lambda}^{g+}}\right|^{2}+\left|\overline{\hat{b}_{\lambda}^{h} \hat{b}_{\lambda}^{g}}\right|^{2}}{2\left(\overline{\hat{b}_{\lambda}^{h+} \hat{b}_{\lambda}^{h}}+1 / 2\right)\left(\overline{\hat{b}_{\lambda}^{g+} \hat{b}_{\lambda}^{g}}+1 / 2\right)}\right]^{1 / 2}
$$

Entangled condition for collinear gluons is

$$
0<\left|t \frac{\alpha_{s} \pi}{\sqrt{2} k_{0}}\left(f_{a h b} f_{a h c}+f_{a g b} f_{a g c}+f_{a h b} f_{a g c}+f_{a g b} f_{a h c}\right) \sum_{\lambda_{1} \neq \lambda}\right| \alpha_{\lambda_{1}}^{b} \| \alpha_{\lambda_{1}}^{c}\left|\sin \left(\gamma_{\lambda_{1}}^{b}+\gamma_{\lambda_{1}}^{c}\right)\right|<1
$$

Obviously squeezed gluon states are simultaneously entangled if the amplitudes of the initial gluon coherent fields are small enough.

Thus by analogy with quantum optics as a result of four-gluon self-interaction we obtain two-mode squeezed gluon states which are also entangled. 


\section{Interaction of quark colour states with QCD vacuum}

We might investigate the dynamics of individual quarks and multi-quark systems, using the similar information-centered approach. The model of stochastic vacuum of QCD is known to be a possible phenomenological solution for the confinement of quarks problem [12-14], has been proven to be applicable by lattice calculations [15] and thus could serve as an effective basis for such an attempt.

While using this model, we consider that only the second field correlators are giving a nonnegligible contribution (Gauss domination) and also use the fact that the interactions of some quantum system with the environment can be effectively represented by additional stochastic terms in the Hamiltonian of the system. Then the density matrix of the system is obtained by averaging with respect to degrees of freedom of the quantum environment - the stochastic vacuum of QCD. Interactions with the environment and the corresponding non-unitary evolution result in decoherence and loss of quantum superpositions. So the information on the initial state of the quantum system (in this case the colour state of quarks) is lost after sufficiently large time.

Let us now consider the interaction of colour particle (quark) with QCD stochastic vacuum. Assume this quark propagates along some fixed path $\gamma$. The amplitude of such process is obtained by [21]

$$
|\phi(\gamma)\rangle=\mathcal{P} \exp \left(i \int_{\gamma} d x^{\mu} \hat{A}_{\mu}\right)\left|\phi_{i n}\right\rangle,
$$

where $\mathcal{P}$ is the path ordering operator, $\hat{A}_{\mu}$ is the gauge field vector and $\left|\phi_{i n}\right\rangle$ is the initial state vector. In order to consider mixed states we introduce the colour density matrix, taking into account both colour particle and QCD stochastic vacuum (environment):

$$
\hat{\rho}(\gamma)=<|\phi(\gamma)\rangle\langle\phi(\gamma)|>
$$

Here we average over all implementations of stochastic gauge field (environment degrees of freedom). In order to keep the gauge invariance, the path $\gamma$ should represent a closed loop. The averaging yields [22]:

$$
\hat{\rho}(\gamma)=N_{c}^{-1}+\left(\left|\phi_{i n}\right\rangle\left\langle\phi_{i n}\right|-N_{c}^{-1}\right) W_{a d j}(\gamma) .
$$

Here $N_{c}$ is the number of colours, $W_{a d j}$ is the Wilson loop in the adjoint representation.

In confinement region Wilson loop decays exponentially with the area spanned by loop, so for the rectangular loop spanning the time interval $T$ and distance $R$ we get:

$$
\begin{gathered}
\hat{\rho}(\gamma)=N_{c}^{-1}+\left(\hat{\rho}_{\text {in }}-N_{c}^{-1}\right) \exp \left(-\sigma_{\text {adj }} R T\right), \\
\hat{\rho}(\gamma: R T \rightarrow \infty)=N_{c}^{-1},
\end{gathered}
$$

where $\sigma_{a d j}$ is the string tension in the adjoint representation.

As follows from (20), all colour states are mixed with equal probabilities and all information on initial color state is lost. The same result might be acquired for the interaction of colour superposition with stochastic QCD vacuum [23].

\section{Quantum optical characteristics}

The rate of transition from pure colour states to white mixture state can be estimated on the base of purity [24]:

$$
P=\operatorname{Tr} \hat{\rho}^{2} .
$$


This characteristic represents the closeness of a quantum state to a pure one. In our case,

$$
P=N_{c}^{-1}+\left(1-N_{c}^{-1}\right) \exp \left(-\sigma_{a d j} R T\right) .
$$

When $R T$ tends to $0, P \rightarrow 1$, which corresponds to a pure state. When the composition $R T$ tends to infinity, the purity tends to $N_{c}^{-1}$, which corresponds to the white mixture.

It can be inferred from (18) and (22) that the larger the loop is, the quicker the initial state tends to white mixture as a result of interaction with the QCD stochastic vacuum. Thus the information of quark colour states is lost in hadrons due to interactions between quarks and confining non-Abelian gauge fields.

Von Neumann entropy can be used as a measure of the loss of information:

$$
S=-\operatorname{Tr}(\hat{\rho} \ln \hat{\rho}) .
$$

Initial density matrix gives us $S=0$ and we will have $S=\ln N_{c}$ for large $R T$.

In order to obtain a more suitable characteristic to work with, we would define the information on the basis of von Neumann entropy. As it can be seen, the entropy ranges from 0 (for a pure, unperturbed quantum state, which in our approach corresponds to the maximum amount of quantum information) to $\ln N_{c}$. So it would be quite natural to define the information measure as

$$
I=\frac{\ln N_{c}-S}{\ln N_{c}}=1-\frac{S}{\ln N_{c}}
$$

So the overall range of this measure is $[0,1]$ : in case of zero entropy it is equal to 1 and in case of maximum entropy it is equal to 0 . The latter case corresponds to the asymptotically big values of $R T$ (the confinement region).

Thus, as we might see, during the interaction with QCD vacuum the entropy increases and the information is being lost.

\section{Multiparticle States and QCD Vacuum}

Density matrix of a system is dependent on the quantity of its degrees of freedom, which is dependent on the number of particles in the system. Accordingly, the density matrix dimesions for multiparticle states would be increased from $\left(N_{c}\right) \mathrm{x}\left(N_{c}\right)$ to $\left(N_{c}^{N_{p}}\right) \mathrm{x}\left(N_{c}^{N_{p}}\right)$, where $N_{p}$ is the number of particles. So the expression (19) in the multiparticle case would look like

$$
\hat{\rho}=N_{c}^{-N_{p}} \hat{I}+\left(\hat{\rho}_{i n}-N_{c}^{-N_{p}} \hat{I}\right) \exp \left(-\sigma_{a d j} R T\right) .
$$

And the expression (20) can be written as

$$
\hat{\rho}(\gamma: R T \rightarrow \infty)=N_{c}^{-N_{p}} \hat{I} .
$$

The purity (21) would change to:

$$
\mathrm{P}=N_{c}^{-N_{p}}+\left(1-N_{c}^{-N_{p}}\right) W_{a d j}^{2}(\gamma),
$$

with maximum von Neumann entropy (23) being:

$$
S=N_{p} \ln N_{c}
$$

The proposed information measure (24) might then be generalized as 
Table 1. Purity and entropy of different states of multiparticle system before the interaction with vacuum

\begin{tabular}{lllll}
\hline State: & pure separable & mixed separable & pure entangled & mixed entangled \\
\hline $\mathrm{P}$ (purity) & 1 & $\frac{1}{N_{c}^{N_{p}}} \leq P<1$ & 1 & $\frac{1}{N_{c}^{N_{p}}}<P<1$ \\
$\mathrm{~S}$ (entropy) & 0 & $0<S \leq N_{p} \ln N_{c}$ & 0 & $0<S<N_{p} \ln N_{c}$ \\
\hline
\end{tabular}

Table 2. Purity and entropy of different states of multiparticle system after the interaction with vacuum

\begin{tabular}{lllll}
\hline State: & pure separable & mixed separable & pure entangled & mixed entangled \\
\hline $\mathrm{P}$ (purity) & $\frac{1}{N_{c}^{N_{p}}}$ & $\frac{1}{N_{c}^{N_{p}}}$ & $\frac{1}{N_{c}^{N_{p}}}$ & $\frac{1}{N_{c}^{N_{p}}}$ \\
$\mathrm{~S}$ (entropy) & $N_{p} \ln N_{c}$ & $N_{p} \ln N_{c}$ & $N_{p} \ln N_{c}$ & $N_{p} \ln N_{c}$ \\
\hline
\end{tabular}

$$
I=1-\frac{S}{N_{p} \ln N_{c}}
$$

Here is the list of possible types of states, in which the system may be found (excluding some specific types e.g. as the squeezed states):

- By purity: 1) pure; 2) mixed.

- By separability: 1) separable; 2) non-separable (entangled).

Table 1 represents the quantum characteristics for the four types of states (before the interaction with vacuum). The strictness of the inequalities in the last column is due to the fact that a fully mixed state is always separable.

The same quantities after the interaction are shown in table 2.

Thus we can see that the final state is defined by a number of particles (and the number of possible colours for any individual particle) in the system. But in any case the entropy during the interaction with QCD vacuum rises to its maximum value and the information is being lost in this process. In the limit $W(\gamma) \rightarrow 0$ we get fully mixed state of a form (26).

\section{Conclusion}

Investigating of the gluon fluctuations we have proved theoretically the possibility of existence of the multimode gluon squeezed states. The emergence of such remarkable states becomes possible owing to the four-gluon self-interaction. The three-gluon self-interaction does not lead to squeezing effect.

We have shown that QCD jet non-perturbative evolution leads both to squeezing and entanglement of gluons. It should be noted that the greater are both the amplitudes of the initial gluon coherent fields with different colour and polarization indexes and coupling constant, the greater is multimode squeezing effect of the colour gluons. We have demonstrated that entanglement condition of the gluon states with fixed two colours and polarization is defined by the corresponding squeeze factor which is dependent on the amplitudes and phases of initial coherent gluon fields.

Because two-mode gluon states with two different colours can lead to $q \bar{q}$-entangled states role of colour correlations could be very significant for explanation of the hadronization phenomenon.

Vacuum of quantum chromodynamics can be considered as environment for colour particles. Density matrix, purity and von Neumann entropy for colour particles depend on Wilson loop averaged through QCD vacuum degrees of freedom. The interaction of colour particle with QCD vacuum leads to the decoherence of pure colour states into a mixed white states. 
For multiparticle states (pure separable, mixed separable and entangled) in the asymptotic of large time and space intervals we obtain diagonalization of density matrix, decreasing of purity and increasing of von Neumann entropy.

\section{References}

[1] Yu.L. Dokshitzer, V.A. Khoze, A.H. Mueller, S. I. Troyan, Basics of Perturbative QCD (Frontières, France, 1991)

[2] V.I. Kuvshinov, Acta Phys. Pol. B 10, 19 (1979)

[3] E.S. Kokoulina, V.I. Kuvshinov, Acta Phys. Pol. B 13, 553 (1982)

[4] D.F. Walls, G.J. Milburn, Quantum Optics (Springer-Verlag, NY., USA, 1995)

[5] M.O. Scully, M.S. Zubairy, Quantum Optics (Cambridge University Press, 1997)

[6] S.Ya. Kilin, Quantum Optics (Minsk, 1990). (in Russian)

[7] I.M. Dremin, Phys. Lett. A 193, 209 (1994)

[8] S. Lupia, W. Ochs and J. Wosiek, Nucl. Phys. B 540, 405 (1999)

[9] G.J. Alner et al. (UA5 coll.), Phys. Rep. 154, 247 (1987)

[10] P. Abreu et al. (DELPHI coll.), Z. Phys. C 50, 185 (1991)

[11] P.D. Acton et al. (OPAL coll.), Z. Phys. C 53, 539 (1992)

[12] Yu. A. Simonov. The Confinement. Phys. Usp. 39, 313 (1996).

[13] J. Ambjørn, P. Olesen. On the Formation of a Color Magnetic Quantum Liquid in QCD. Nuclear Physics B. 170, no. 1, 60-78 (1980).

[14] D. S. Kuzmenko, Y. A. Simonov, et al. Vacuum, Confinement and QCD Strings in the Vacuum Correlator Method. Uspekhi Fizicheskih Nauk. 174, no. 1 (2004) (in Russian).

[15] A. D. Giacomo, H. Dosch, V. I. Shevchenko, and Y. A. Simonov, Physics Reports 372, no. 4, 319-368 (2002).

[16] J.S. Bell, Physics 1, 195 (1964)

[17] A. Einstein, B. Podolsky and N. Rosen, Phys. Rev. 45, 777 (1935)

[18] E.A. De Wolf, Progress in Optics. 42, 1 (2001)

[19] V.V. Dodonov, A.S.M. de Castro, S.S. Mizrahi, Phys. Lett. A 296, 73 (2002)

[20] V.I. Kuvshinov, V.A. Shaparau, Ukrainian Journal of Physics 48, 1255 (2003)

[21] V. Kuvshinov, P. Buividovich. White Mixed States in QCD Stochastic Vacuum. Nonlinear Phenomena in Complex Systems. 8, no.3, 313-316 (2005).

[22] V. Kuvshinov, A. Kuzmin. Gauge Fields and Theory of Deterministic Chaos. (Belorussian Science, Minsk, 2006, in Russian).

[23] V. I. Kuvshinov, E. G. Bagashov. Evolution of colour superposition in the stochastic QCD vacuum. Nonlinear Phenom. Complex Syst., 16, No. 3, 242-246 (2013).

[24] F. Haake. Quantum Signatures of Chaos. (Springer-Verlag, Berlin, 1991). 